Article

\title{
Insecticide Resistance Monitoring in Field Populations of the Whitebacked Planthopper Sogatella furcifera (Horvath) in China, 2019-2020
}

\author{
Zhao Li $^{\dagger}$, Yao Qin ${ }^{\dagger}$, Ruoheng Jin, Yunhua Zhang, Zhijie Ren, Tingwei Cai ${ }^{\circledR}$, Chang Yu, Yu Liu, Yongfeng Cai, \\ Qinghong Zeng, Hu Wan and Jianhong $\mathrm{Li}^{*}$ *
}

check for

updates

Citation: Li, Z.; Qin, Y.; Jin, R.; Zhang, Y.; Ren, Z.; Cai, T.; Yu, C.; Liu, Y.; Cai, Y.; Zeng, Q.; et al. Insecticide Resistance Monitoring in Field Populations of the Whitebacked Planthopper Sogatella furcifera (Horvath) in China, 2019-2020. Insects 2021, 12, 1078. https://doi.org/ $10.3390 /$ insects 12121078

Academic Editor: Emmanouil Roditakis

Received: 9 September 2021

Accepted: 29 November 2021

Published: 30 November 2021

Publisher's Note: MDPI stays neutral with regard to jurisdictional claims in published maps and institutional affiliations.

Copyright: (c) 2021 by the authors. Licensee MDPI, Basel, Switzerland. This article is an open access article distributed under the terms and conditions of the Creative Commons Attribution (CC BY) license (https:// creativecommons.org/licenses/by/ $4.0 /)$.
Hubei Insect Resources Utilization and Sustainable Pest Management Key Laboratory, College of Plant Science and Technology, Huazhong Agricultural University, Wuhan 430070, China; Lucky_edmund@163.com (Z.L.); qinyao1246282025@163.com (Y.Q.); jinshirley@126.com (R.J.); zhangyunh6688@126.com (Y.Z.); rzj@webmail.hzau.edu.cn (Z.R.); caitingwei@webmail.hzau.edu.cn (T.C.); ycliule@163.com (C.Y.); 15071403650@163.com (Y.L.); yongfengcai1996@163.com (Y.C.); zqh854597499@163.com (Q.Z.); huwan@mail.hzau.edu.cn (H.W.)

* Correspondence: jianhl@mail.hzau.edu.cn; Tel./Fax: +86-27-8728-6968

+ These authors contributed equally to this paper.

Simple Summary: The whitebacked planthopper (WBPH), Sogatella furcifera (Horváth), is one of the most destructive pests that seriously threatens the high-quality and safe production of rice. Overuse of chemical insecticides has led to varying levels of resistance to insecticides in the field population of S. furcifera. In this study, we measured the susceptibility of 18 populations to 10 insecticides by the rice-seedling dip method. Enzyme assays were performed to measure the levels of esterase (EST), glutathione $S$ - transferase (GST) and cytochrome P450 monooxygenase (P450). A risk of cross-resistance between some insecticides were found by pairwise correlation, and EST may be contributed to the resistance to nitenpyram, thiamethoxam and clothianidin in S. furcifera. Overall, our findings will help inform the effective insecticide resistance management strategies to delay the development of insecticide resistance in S. furcifera.

Abstract: Monitoring is an important component of insecticide resistance management. In this study, resistance monitoring was conducted on 18 field populations in China. The results showed that S. furcifera developed high levels of resistance to chlorpyrifos and buprofezin, and S. furcifera showed low to moderate levels of resistance to imidacloprid, thiamethoxam, dinotefuran, clothianidin, sulfoxaflor, isoprocarb and ethofenprox. Sogatella furcifera remained susceptible or low levels of resistance to nitenpyram. $\mathrm{LC}_{50}$ values of nitenpyram and dinotefuran, imidacloprid, thiamethoxam, clothianidin and chlorpyrifos exhibited significant correlations, as did those between dinotefuran and thiamethoxam, clothianidin, sulfoxaflor, imidacloprid, isoprocarb and buprofezin. Similarly, significant correlations were observed between thiamethoxam and clothianidin, sulfoxaflor and imidacloprid. In addition, the activity of EST in field populations of S. furcifera were significantly correlated with the $\mathrm{LC}_{50}$ values of nitenpyram, thiamethoxam and clothianidin. These results will help inform effective insecticide resistance management strategies to delay the development of insecticide resistance in $S$. furcifera.

Keywords: Sogatella furcifera; insecticide; insecticide resistance; detoxification enzymes; correlation analysis

\section{Introduction}

The whitebacked planthopper (WBPH), Sogatella furcifera (Horváth: Delphacidae, Sogatella), is one of the most destructive pests of rice crops and is widely distributed in Asian countries [1,2], causing serious damage to rice mainly by sucking at the base of rice stems and spreading viral diseases [3,4]. Chemical insecticides have a long history of controlling the $\mathrm{WBPH}$, and they are still the primary measure of prevention and management $[2,5,6]$. 
However, due to long-term use of synthetic insecticides, various populations of WBPH have developed resistance to 15 compounds worldwide, including organophosphates, pyrethroids, carbamates, insect growth regulators and neonicotinoids [7-9]. Consequently, it is important to determine the resistance level of the field population of WBPH to frequently used insecticides, which are crucial for the successful management of the WBPH.

Previous studies have demonstrated an altered target site and enhanced detoxification (metabolism) as the main mechanisms of resistance to insecticides [10-12]. In S. furcifera, metabolic resistance due to overexpression of cytochrome P450 (monooxygenase) has contributed to neonicotinoid, insect growth regulator and organophosphate resistance, and an elevated level of GST has also been involved in resistance to neonicotinoids and insect growth regulator resistance $[13,14]$. In addition, increasing activities of EST have contributed to insect growth regulator resistance in S. furcifera [15].

The WBPH has become increasingly damaging in rice producing areas in the Yangtze River region, which is the main paddy field in China [8]. In our study, we assessed the resistance situation of $S$. furcifera to 10 insecticides (including carbamates, pyrethroids, neonicotinoids, organophosphates and insect growth regulators) from 18 areas of six provinces in China, and analyzed the potential cross-resistance patterns of these insecticides in all collected field populations of $S$. furcifera. We also determined the correlation between the activity of detoxification enzymes and the susceptibilities of $S$. furcifera field populations. The findings of this study not only provide directions for the sustainable control of this important pest in rice producing areas, but also provide useful data for the insecticide resistance management and integrated pest management (IPM).

\section{Materials and Methods}

\subsection{Insect Populations}

Eighteen field populations of WBPH were collected from rice paddy fields in six provinces in China (Table 1). Insects were reared under the following conditions: temperature of $27 \pm 1{ }^{\circ} \mathrm{C}$, relative humidity (RH) of $70-80 \%$ and photoperiod of $16: 8 \mathrm{~h} \mathrm{light/dark.}$ The field-collected WBPH was mass mated and maintained in the laboratory for 1-2 generations, and the progeny were used for the susceptibility bioassay and enzyme assays.

\subsection{Insecticides}

The 10 insecticides were sourced and tested: nitenpyram (Hubei Kangbaotai FineChemicals Co., Ltd., Wuhan, China, 95.8\%), dinotefuran (Hubei Kangbaotai Fine-Chemicals Co., Ltd., 91.0\%), thiamethoxam (Hubei Kangbaotai Fine-Chemicals Co., Ltd., 95\%), clothianidin (Hubei Kangbaotai Fine-Chemicals Co., Ltd., Wuhan, China, 96.0\%), sulfoxaflor (Dow AgroSciences Inc., Shanghai, China, 97.9\%), imidacloprid (Hubei Kangbaotai FineChemicals Co., Ltd., Wuhan, China, 95.8\%), chlorpyrifos (Hebei VeYong Bio-Chemical Co., Ltd., Shijiazhuang, China, 98.0\%), isoprocarb (Jiangsu Changlong Chemicals Co., Ltd., Changzhou, China, 97.9\%), ethofenprox (Suzhou ATL Chemical Co., Ltd., Suzhou, China, 95.0\%) and buprofezin (Jiangsu Anpon Electrochemical Co., Ltd., Huai'an, China, 97.4\%). The modes of action included nitenpyram, dinotefuran, thiamethoxam, clothianidin, sulfoxaflor and imidacloprid via nicotinic acetylcholine receptor (nAChR) competitive modulators; chlorpyrifos and isoprocarb via acetylcholinesterase (AChE) inhibitors; ethofenprox and buprofezin via sodium channel modulators and inhibitors of chitin biosynthesis, respectively [16].

\subsection{Bioassays}

Bioassays were performed with third-instar nymphs of $S$. furcifera using a rice-seedling dip method that has been described previously [17]. Briefly, fifteen rice seedlings were combined, immersed in a concentration of insecticide dilutions (Table S1) for $30 \mathrm{~s}$ and then air-dried at $25^{\circ} \mathrm{C}$ for more than $30 \mathrm{~min}$. The roots of rice seedlings were wrapped with cotton moistened with water, and placed in a $500-\mathrm{mL}$ plastic cup. Each replicate contained fifteen third-instar nymphs. Three replicates of each concentration and six replicates of 
each insecticide were carried out, with rice seedlings dipped in water containing $0.1 \%$ Triton X-100 (Sigma-Aldrich, St. Louis, MO, USA). Triton X-100 was used as a control. Mortality was counted after exposure to isoprocarb, chlorpyrifos, and ethofenprox for $72 \mathrm{~h}$, imidacloprid, thiamethoxam, dinotefuran, clothianidin, sulfoxaflor, and nitenpyram for $96 \mathrm{~h}$, and buprofezin for $120 \mathrm{~h}$.

Table 1. Information of $S$. furcifera collected from rice paddy fields of China.

\begin{tabular}{|c|c|c|c|c|c|}
\hline Populations & Sampling Sites & Collection Date & Site & Insect Stage & Number \\
\hline HBCB-2019 & Chibi, Hubei & 15 July 2019 & $29^{\circ} 31^{\prime} \mathrm{N}, 113^{\circ} 42^{\prime} \mathrm{E}$ & Nymph and adult & 1206 \\
\hline HBWX-2019 & Wuxue, Hubei & 6 September 2019 & $29^{\circ} 57^{\prime} \mathrm{N}, 115^{\circ} 36^{\prime} \mathrm{E}$ & Nymph and adult & 1374 \\
\hline НВJM-2019 & Jingmen, Hubei & 5 September 2019 & $30^{\circ} 32^{\prime} \mathrm{N}, 112^{\circ} 18^{\prime} \mathrm{E}$ & Nymph and adult & 1453 \\
\hline HNNX-2019 & Ningxiang, Hunan & 20 June 2019 & $28^{\circ} 10^{\prime} \mathrm{N}, 112^{\circ} 33^{\prime} \mathrm{E}$ & Nymph and adult & 1069 \\
\hline JXNC-2019 & Nanchang, Jiangxi & 12 June 2019 & $28^{\circ} 32^{\prime} \mathrm{N}, 115^{\circ} 58^{\prime} \mathrm{E}$ & Nymph and adult & 1146 \\
\hline AHLA-2019 & Luan, Anhui & 10 July 2019 & $31^{\circ} 32^{\prime} \mathrm{N}, 116^{\circ} 18^{\prime} \mathrm{E}$ & Nymph and adult & 1073 \\
\hline HNXY-2019 & Xinyang, Henan & 14 July 2019 & $31^{\circ} 58^{\prime} \mathrm{N}, 115^{\circ} 24^{\prime} \mathrm{E}$ & Nymph and adult & 1421 \\
\hline HBDY-2020 & Dangyang, Hubei & 19 July 2020 & $30^{\circ} 59^{\prime} \mathrm{N}, 111^{\circ} 52^{\prime} \mathrm{E}$ & Nymph and adult & 1306 \\
\hline HBCB-2020 & Chibi, Hubei & 21 July 2020 & $29^{\circ} 31^{\prime} \mathrm{N}, 113^{\circ} 42^{\prime} \mathrm{E}$ & Nymph and adult & 1154 \\
\hline HBWX-2020 & Wuxue, Hubei & 8 August 2020 & $30^{\circ} 07^{\prime} \mathrm{N}, 115^{\circ} 36^{\prime} \mathrm{E}$ & Nymph and adult & 1073 \\
\hline HBJL-2020 & Jianli, Hubei & 25 July 2020 & $29^{\circ} 49^{\prime} \mathrm{N}, 112^{\circ} 54^{\prime} \mathrm{E}$ & Nymph and adult & 1209 \\
\hline HВJM-2020 & Jingmen, Hubei & 14 July 2020 & $30^{\circ} 54^{\prime} \mathrm{N}, 112^{\circ} 14^{\prime} \mathrm{E}$ & Nymph and adult & 1043 \\
\hline HBXZ-2020 & Xinzhou, Hubei & 14 July 2020 & $30^{\circ} 51^{\prime} \mathrm{N}, 114^{\circ} 37^{\prime} \mathrm{E}$ & Nymph and adult & 1194 \\
\hline HNNX-2020 & Ningxiang, Hunan & 3 August 2020 & $28^{\circ} 10^{\prime} \mathrm{N}, 112^{\circ} 33^{\prime} \mathrm{E}$ & Nymph and adult & 1206 \\
\hline JXNC-2020 & Nanchang, Jiangxi & 25 July 2020 & $28^{\circ} 32^{\prime} \mathrm{N}, 115^{\circ} 58^{\prime} \mathrm{E}$ & Nymph and adult & 1311 \\
\hline AHHF-2020 & Luan, Anhui & 12 August 2020 & $31^{\circ} 31^{\prime} \mathrm{N}, 116^{\circ} 37^{\prime} \mathrm{E}$ & Nymph and adult & 1248 \\
\hline HNXY-2020 & Xinyang, Henan & 15 August 2020 & $31^{\circ} 58^{\prime} \mathrm{N}, 115^{\circ} 24^{\prime} \mathrm{E}$ & Nymph and adult & 1076 \\
\hline GZQN-2020 & Qiannan, Guizhou & 15 August 2020 & $25^{\circ} 59^{\prime} \mathrm{N}, 106^{\circ} 35^{\prime} \mathrm{E}$ & Nymph and adult & 1108 \\
\hline
\end{tabular}

\subsection{Enzyme Assays}

EST activity was determined using the method described previously [7]. Approximately $0.02 \mathrm{~g}$ of third-instar nymphs for each repetition was homogenized in $1 \mathrm{~mL}$ of ice-cold sodium phosphate buffer $(0.04 \mathrm{M}, \mathrm{pH} 7.0)$ and centrifuged at $4{ }^{\circ} \mathrm{C}$ and $14,000 \mathrm{rpm}$ for $10 \mathrm{~min}$. The homogenized supernatant was then carefully transported to a new Eppendorf tube and used as the enzyme source. A total of $1.2 \mathrm{~mL}$ of substrate solution (containing $1 \mathrm{~mL} 3 \times 10^{-4} \mathrm{M} \alpha$-NA and $0.2 \mathrm{~mL}$ of the enzyme source) was added and then incubated at $37^{\circ} \mathrm{C}$ for $15 \mathrm{~min}$. The reaction was terminated by the addition of $0.2 \mathrm{~mL}$ of the dye reagent. An NP80 nanophotometer (IMPLEN, Munich, Germany) was used to measure the optical density at $600 \mathrm{~nm}$.

GST activity was assayed as previously described [7]. The enzyme solution was prepared as it was in the EST assay. For each reaction, $740 \mu \mathrm{L}$ of sodium phosphate buffer (0.1 M, pH 6.5), and $30 \mu \mathrm{L}$ of $30 \mathrm{mM}$ 1-chloro-2,4-dinitrobenzene (CDNB), $100 \mu \mathrm{L}$ of enzyme solution, $30 \mu \mathrm{L}$ of $30 \mathrm{mM}$ GSH were mixed. The absorbance was recorded using an NP80 NanoPhotometer (IMPLEN, Munich, Germany) at $340 \mathrm{~nm}$ for $2 \mathrm{~min}$.

The 7-ethoxycoumarin-O-deethylase (7-ECOD) activity of P450 was determined as described previously with slight modification [18]. Approximately $0.2 \mathrm{~g}$ of the thirdinstar nymphs was homogenized in $1.0 \mathrm{~mL}$ of $0.1 \mathrm{M}$ sodium phosphate buffer containing $1 \mathrm{mM}$ ethylenediaminetetraacetic acid EDTA (Sinopharm Chemical Reagent Co., Ltd., Shanghai, China) $1 \mathrm{mM}$ phenylmethylsulfonyl fluoride (PMSF), $1 \mathrm{mM}$ dithiothreitol (DTT) and $10 \%$ glycerol, and then centrifuged at $4{ }^{\circ} \mathrm{C} 14,000 \mathrm{rpm}$ for $15 \mathrm{~min}$. The supernatant was centrifuged at $4{ }^{\circ} \mathrm{C} 14,000 \mathrm{rpm}$ for $30 \mathrm{~min}$ again. For the reaction, $125 \mu \mathrm{L}$ of crude homogenate was mixed with $365 \mu \mathrm{L}$ of $0.1 \mathrm{M}$ sodium phosphate buffer ( $\mathrm{pH} 7.5), 10 \mu \mathrm{L}$ of $10 \mathrm{mM}$ aqueous NADPH and $5 \mu \mathrm{L}$ of $40 \mathrm{mM}$ 7-ECOD and then incubated at $30{ }^{\circ} \mathrm{C}$ for $15 \mathrm{~min}$. The reaction was terminated by adding $150 \mu \mathrm{L}$ of $15 \%$ trichloroacetic acid (TCA). Then, the mixture was centrifuged, and $1.6 \mathrm{mM}$ glycine- $\mathrm{NaOH}$ buffer ( $\mathrm{pH} 10.5)$ was added. A Spark 10 M Multimode Microplate Reader (Tecan, Männedorf, Switzerland) was used for measuring the fluorescence intensity with an excitation wavelength of $358 \mathrm{~nm}$ 
and an emission wavelength of $465 \mathrm{~nm}$. The protein content of the enzyme solutions was determined using Quick Start ${ }^{\mathrm{TM}}$ Bradford ${ }^{*}$ Dye Reagent (Bio-Rad Laboratories, Inc., Hercules, CA, USA).

\subsection{Data Analyses}

Abbott's formula was used to control mortality for the mortality data obtained [19]. The slopes with standard errors (SE), and $\mathrm{LC}_{50}$ values with $95 \%$ confidence intervals, were calculated by probit analysis. The $\mathrm{LC}_{50}$ of the most susceptible population was confirmed in previous studies in our lab [7]. The classification standard of resistance level was based on the "Technological rules for monitoring insecticide resistance in rice white-backed planthopper, Sogatella furcifera" (NY/T 3159-2017), Agricultural industry standard of the people's Republic of China. The resistance ratio (RR): $R R \leq 5,5<R R \leq 10,10<R R \leq$ 100 and RR $>100$ were classified as susceptible, low level of resistance, moderate level of resistance, and high level of resistance, respectively. The $\mathrm{LC}_{50}$ values of the susceptibility baseline and minimum recommended dose of registered insecticides to $S$. furcifera are listed in Table 2. Spearman correlations between the $\mathrm{LC}_{50}$ values of different insecticides were calculated using the WGCNA package (version 1.69) and visualized using corrplot (version 0.1.3). The relative enzyme activities and differences in mortality were analyzed using unpaired Student's t-tests with at least three repeats. The standard of statistically significant differences was $p<0.05$.

Table 2. The susceptibility baseline of $S$. furcifera to insecticides.

\begin{tabular}{|c|c|c|c|}
\hline Group & Insecticide & $\mathrm{LC}_{50}\left(95 \% \mathrm{CI}^{\mathrm{a}}\right)(\mathrm{mg} / \mathrm{L})$ & Reference \\
\hline \multirow{5}{*}{ neonicotinoids } & imidacloprid & $0.11(0.06-0.17)$ & Su et al., 2013 \\
\hline & thiamethoxam & $0.18(0.11-0.23)$ & Su et al., 2013 \\
\hline & nitenpyram & $0.27(0.19-0.36)$ & Zhang et al., 2017 \\
\hline & dinotefuran & $0.20(0.16-0.25)$ & Li et al., 2020 \\
\hline & clothianidin & $0.15(0.09-0.21)$ & Zhang et al., 2017 \\
\hline Sulfoximines & sulfoxaflor & $0.50(0.33-0.66)$ & Li et al., 2020 \\
\hline Insect growth regulators & buprofezin & $0.04(0.03-0.06)$ & Li et al., 2020 \\
\hline Carbamates & isoprocarb & $9.42(6.97-11.80)$ & Li et al., 2020 \\
\hline Pyrethroids & ethofenprox & $34.61(20.28-52.76)$ & Li et al., 2020 \\
\hline Organophosphates & chlorpyrifos & $0.24(0.17-0.31)$ & Li et al., 2020 \\
\hline
\end{tabular}

\section{Results}

\subsection{Insecticide Resistance}

To determine the resistance level of S. furcifera in rice paddy fields in China, we measured the susceptibility of 18 populations to 10 insecticides by the rice-seedling dip method. The results indicated that $S$. furcifera had moderate to high levels of resistance to chlorpyrifos $(R R=47.75-304.17$-fold $)$ and buprofezin $(R R=81.25-331.50$ fold). Furthermore, $S$. furcifera developed low to moderate resistance levels to dinotefuran $(\mathrm{RR}=1.35-51.45$-fold $)$, thiamethoxam $(\mathrm{RR}=1.33-16.61$-fold $)$, clothianidin $(\mathrm{RR}=1.80-17.33$ fold), sulfoxaflor ( $R R=4.70-32.26$-fold), imidacloprid ( $R R=2.09-62.55$-fold), isoprocarb $(\mathrm{RR}=2.78-22.85$-fold $)$ and ethofenprox $(\mathrm{RR}=6.06-19.62$-fold $)$. In addition, the resistance level of $S$. furcifera to nitenpyram ( $R R=0.67-9.67$-fold) remained susceptible to low levels (Table 3, Figure 1). 


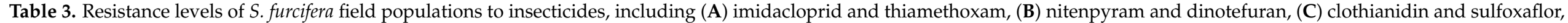

(D) buprofezin and chlorpyrifos, (E) isoprocarb and ethofenprox.

\begin{tabular}{|c|c|c|c|c|c|c|c|c|c|c|c|c|}
\hline \multicolumn{13}{|c|}{ A } \\
\hline \multirow{2}{*}{ Population } & \multicolumn{6}{|c|}{ Imidacloprid } & \multicolumn{6}{|c|}{ Thiamethoxam } \\
\hline & No. & Slope (SE $\left.{ }^{a}\right)$ & $\mathrm{LC}_{50}\left(95 \% \mathrm{CI}^{\mathrm{b}} ; \mathrm{mg} / \mathrm{L}\right)$ & $\chi^{2}(\mathrm{df})$ & $p$ Value & $\mathbf{R R}^{\mathrm{c}}$ & No. & Slope (SE $\left.{ }^{a}\right)$ & $\mathrm{LC}_{50}\left(95 \% \mathrm{CI}^{\mathrm{b}} ; \mathrm{mg} / \mathrm{L}\right)$ & $\chi^{2}(\mathrm{df})$ & $p$ Value & $\mathbf{R R}^{\mathrm{c}}$ \\
\hline НВCВ-2019 & 270 & $2.00(0.27)$ & $0.72(0.49-0.94)$ & $0.67(3)$ & 0.88 & 6.55 & 270 & $1.53(0.23)$ & $1.31(0.99-1.90)$ & $5.62(3)$ & 0.13 & 7.28 \\
\hline HBWX-2019 & 270 & $1.88(0.25)$ & $0.23(0.17-0.30)$ & $0.89(3)$ & 0.83 & 2.09 & 270 & $1.64(0.23)$ & $0.24(0.17-0.32)$ & $3.08(3)$ & 0.38 & 1.33 \\
\hline HВJM-2019 & 270 & $2.32(0.27)$ & $1.43(1.14-1.74)$ & $1.11(3)$ & 0.77 & 13.00 & 270 & $1.67(0.23)$ & $0.98(0.77-1.30)$ & $0.69(3)$ & 0.88 & 5.44 \\
\hline HNNX-2019 & 270 & $1.72(0.24)$ & $1.63(1.11-2.14)$ & $0.82(3)$ & 0.84 & 14.82 & 270 & $2.02(0.25)$ & $1.51(1.21-1.87)$ & $4.37(3)$ & 0.22 & 8.39 \\
\hline JXNC-2019 & 270 & $1.90(0.28)$ & $2.28(1.66-3.82)$ & $1.63(3)$ & 0.65 & 20.73 & 270 & $1.62(0.25)$ & $2.08(1.50-3.50)$ & $1.84(3)$ & 0.61 & 11.56 \\
\hline AHLA-2019 & 270 & $2.48(0.28)$ & $2.67(2.19-3.21)$ & $2.16(3)$ & 0.54 & 24.27 & 270 & $1.70(0.24)$ & $2.99(2.29-4.35)$ & $4.08(3)$ & 0.25 & 16.61 \\
\hline HNXY-2019 & 270 & $1.65(0.23)$ & $1.46(1.08-1.88)$ & $2.04(3)$ & 0.56 & 13.27 & 270 & $1.54(0.24)$ & $1.96(1.41-3.29)$ & $1.17(3)$ & 0.11 & 10.88 \\
\hline HBDY-2020 & 270 & $1.68(0.23)$ & $1.19(0.92-1.63)$ & $1.78(3)$ & 0.58 & 10.82 & 270 & $1.81(0.24)$ & $1.07(0.79-1.35)$ & $0.95(3)$ & 0.81 & 5.94 \\
\hline HBCB-2020 & 270 & $1.30(0.22)$ & $1.88(1.37-2.66)$ & $2.28(3)$ & 0.19 & 17.09 & 270 & $1.30(0.22)$ & $1.17(0.86-1.75)$ & $3.61(3)$ & 0.31 & 6.50 \\
\hline HBWX-2020 & 270 & $0.82(0.21)$ & $1.40(0.77-6.04)$ & $3.20(3)$ & 0.86 & 12.73 & 270 & $1.17(0.21)$ & $0.42(0.30-0.61)$ & $2.29(3)$ & 0.51 & 2.33 \\
\hline HBJL-2020 & 270 & $1.17(0.21)$ & $2.94(2.09-4.61)$ & $3.72(3)$ & 0.84 & 26.73 & 270 & $1.84(0.26)$ & $1.56(1.17-2.33)$ & $3.54(2)$ & 0.17 & 8.67 \\
\hline HBJM-2020 & 270 & $2.33(0.27)$ & $2.38(1.97-2.94)$ & $2.45(3)$ & 0.87 & 21.64 & 270 & $1.86(0.25)$ & $2.53(2.00-3.43)$ & $1.31(3)$ & 0.73 & 14.06 \\
\hline HBXZ-2020 & 270 & $1.32(0.22)$ & $2.28(1.68-3.20)$ & 0.79 (3) & 0.42 & 20.73 & 270 & $1.27(0.22)$ & $3.22(2.29-5.46)$ & $3.73(3)$ & 0.29 & 17.89 \\
\hline HNNX-2020 & 270 & $1.43(0.22)$ & $3.25(2.43-4.82)$ & $2.44(3)$ & 0.40 & 29.55 & 270 & $2.08(0.25)$ & $1.50(1.21-1.85)$ & $3.06(3)$ & 0.38 & 8.33 \\
\hline JXNC-2020 & 270 & $2.13(0.25)$ & $2.02(1.64-2.49)$ & $0.57(3)$ & 0.61 & 18.36 & 270 & $2.10(0.25)$ & $1.79(1.45-2.22)$ & $3.09(3)$ & 0.38 & 9.94 \\
\hline AHHF-2020 & 270 & $1.58(0.26)$ & 6.88 (4.64-13.67) & $2.18(3)$ & 0.80 & 62.55 & 270 & $2.20(0.26)$ & $2.13(1.74-2.69)$ & $0.31(3)$ & 0.96 & 11.83 \\
\hline HNXY-2020 & 270 & $2.58(0.31)$ & $2.21(1.68-2.71)$ & $1.59(3)$ & 0.29 & 20.09 & 270 & $1.84(0.26)$ & $0.90(0.60-1.19)$ & $2.09(3)$ & 0.55 & 5.00 \\
\hline GZQN-2020 & 270 & $2.35(0.29)$ & $3.86(3.09-5.24)$ & $3.83(3)$ & 0.21 & 35.09 & 270 & $1.70(0.28)$ & $3.01(2.01-6.19)$ & $1.88(3)$ & 0.60 & 16.72 \\
\hline \multicolumn{13}{|c|}{ B } \\
\hline \multirow{2}{*}{ Population } & \multicolumn{6}{|c|}{ Nitenpyram } & \multicolumn{6}{|c|}{ Dinotefuran } \\
\hline & No. & Slope (SE $\left.{ }^{\text {a }}\right)$ & $\mathrm{LC}_{50}\left(95 \% \mathrm{CI}^{\mathrm{b}} ; \mathrm{mg} / \mathrm{L}\right)$ & $\chi^{2}(\mathrm{df})$ & $p$ Value & $\mathbf{R R}^{\mathrm{c}}$ & No. & Slope $\left(\mathrm{SE}^{\mathrm{a}}\right)$ & $\mathrm{LC}_{50}\left(95 \% \mathrm{CI}^{\mathrm{b}} ; \mathrm{mg} / \mathrm{L}\right)$ & $\chi^{2}(\mathrm{df})$ & $p$ Value & $\mathbf{R R}^{\mathrm{c}}$ \\
\hline HBCВ-2019 & 270 & $1.56(0.23)$ & $0.67(0.51-0.96)$ & $3.34(3)$ & 0.34 & 2.48 & 270 & $2.12(0.25)$ & $1.92(1.55-2.36)$ & $0.57(3)$ & 0.90 & 9.60 \\
\hline HBWX-2019 & 270 & $1.88(0.24)$ & $0.27(0.20-0.34)$ & $3.51(3)$ & 0.32 & 1.00 & 270 & $2.25(0.27)$ & $0.27(0.21-0.33)$ & $1.31(3)$ & 0.73 & 1.35 \\
\hline HBJM-2019 & 270 & $1.56(0.26)$ & $0.12(0.06-0.17)$ & $2.47(3)$ & 0.48 & 0.67 & 270 & $2.14(0.28)$ & $1.00(0.71-1.27)$ & $0.32(3)$ & 0.96 & 5.00 \\
\hline HNNX-2019 & 270 & $2.79(0.30)$ & $1.44(1.20-1.70)$ & $2.02(3)$ & 0.57 & 5.33 & 270 & $2.76(0.31)$ & $2.70(2.27-3.30)$ & $0.31(3)$ & 0.96 & 13.50 \\
\hline JXNC-2019 & 270 & $2.35(0.27)$ & $1.05(0.87-1.32)$ & $0.04(3)$ & 0.99 & 3.89 & 270 & $1.69(0.25)$ & $4.75(3.50-7.56)$ & $1.14(3)$ & 0.77 & 23.75 \\
\hline AHLA-2019 & 270 & $1.85(0.24)$ & $1.79(1.42-2.28)$ & $2.00(3)$ & 0.57 & 6.63 & 270 & $2.03(0.25)$ & $2.13(1.62-2.65)$ & $1.05(3)$ & 0.79 & 10.65 \\
\hline HNXY-2019 & 270 & $1.70(0.24)$ & $2.61(2.02-3.64)$ & $4.10(3)$ & 0.25 & 9.67 & 270 & $2.72(0.37)$ & $1.28(0.87-1.65)$ & 1.59 (3) & 0.66 & 6.40 \\
\hline HBDY-2020 & 270 & $2.00(0.33)$ & $0.41(0.20-0.61)$ & $1.78(3)$ & 0.62 & 1.52 & 270 & $2.10(0.25)$ & $1.93(1.56-2.38)$ & $3.33(3)$ & 0.34 & 9.65 \\
\hline HBCB-2020 & 270 & $2.03(0.25)$ & $1.08(0.83-1.35)$ & $2.28(3)$ & 0.52 & 4.00 & 270 & $2.12(0.28)$ & $1.76(1.20-2.29)$ & $5.24(3)$ & 0.16 & 8.80 \\
\hline HBWX-2020 & 270 & $2.75(0.31)$ & $0.59(0.49-0.74)$ & $3.20(3)$ & 0.36 & 2.19 & 270 & $1.58(0.25)$ & $1.16(0.81-2.09)$ & $2.42(3)$ & 0.49 & 5.80 \\
\hline
\end{tabular}


Table 3. Cont.

\begin{tabular}{|c|c|c|c|c|c|c|c|c|c|c|c|c|}
\hline \multicolumn{13}{|c|}{ B } \\
\hline \multirow{2}{*}{ Population } & \multicolumn{6}{|c|}{ Nitenpyram } & \multicolumn{6}{|c|}{ Dinotefuran } \\
\hline & No. & Slope (SE ${ }^{\text {a) }}$ & $\mathrm{LC}_{50}\left(95 \% \mathrm{CI}^{\mathrm{b}} ; \mathrm{mg} / \mathrm{L}\right)$ & $x^{2}(\mathrm{df})$ & $p$ Value & $\mathbf{R R}^{\mathrm{c}}$ & No. & Slope (SE $\left.{ }^{\text {a }}\right)$ & $\mathrm{LC}_{50}\left(95 \% \mathrm{CI}^{\mathrm{b}} ; \mathrm{mg} / \mathrm{L}\right)$ & $x^{2}(\mathrm{df})$ & $p$ Value & $\mathbf{R R}^{\mathrm{c}}$ \\
\hline HBJL-2020 & 270 & $1.73(0.24)$ & $1.48(1.14-2.13)$ & $3.72(3)$ & 0.29 & 5.48 & 270 & $2.06(0.26)$ & $6.30(5.06-8.32)$ & $3.82(3)$ & 0.28 & 31.50 \\
\hline HBJM-2020 & 270 & $1.91(0.24)$ & $1.44(1.13-1.80)$ & $2.45(3)$ & 0.48 & 5.33 & 270 & $1.77(0.24)$ & $3.25(2.54-4.49)$ & $0.62(3)$ & 0.89 & 16.25 \\
\hline HBXZ-2020 & 270 & $1.73(0.23)$ & $2.25(1.76-3.02)$ & $0.79(3)$ & 0.85 & 8.33 & 270 & $2.61(0.32)$ & $3.72(3.02-4.92)$ & $2.99(3)$ & 0.39 & 18.60 \\
\hline HNNX-2020 & 270 & $2.16(0.25)$ & $1.67(1.36-2.06)$ & $2.44(3)$ & 0.49 & 6.19 & 270 & $1.44(0.23)$ & $3.52(2.62-5.33)$ & $2.10(3)$ & 0.55 & 17.60 \\
\hline JXNC-2020 & 270 & $2.03(0.26)$ & $0.98(0.73-1.23)$ & $0.57(3)$ & 0.90 & 3.63 & 270 & $1.62(0.22)$ & $2.10(1.62-2.74)$ & $1.18(3)$ & 0.76 & 10.50 \\
\hline AHHF-2020 & 270 & $2.34(0.27)$ & $1.57(1.29-1.91)$ & $2.18(3)$ & 0.53 & 5.81 & 270 & $1.57(0.24)$ & $3.28(2.48-4.78)$ & $0.90(3)$ & 0.83 & 16.40 \\
\hline HNXY-2020 & 270 & $2.02(0.25)$ & $1.14(0.88-1.42)$ & $1.59(3)$ & 0.67 & 4.22 & 270 & $1.77(0.25)$ & $2.12(1.47-2.76)$ & $2.56(3)$ & 0.47 & 10.60 \\
\hline GZQN-2020 & 270 & $2.33(0.27)$ & $1.02(0.84-1.27)$ & $3.83(3)$ & 0.28 & 3.78 & 270 & $2.15(0.30)$ & $10.29(7.76-15.90)$ & $1.80(3)$ & 0.62 & 51.45 \\
\hline \multicolumn{13}{|c|}{$\mathrm{C}$} \\
\hline \multirow{2}{*}{ Population } & \multicolumn{6}{|c|}{ Clothianidin } & \multicolumn{6}{|c|}{ Sulfoxaflor } \\
\hline & No. & Slope $\left(\mathrm{SE}^{\mathrm{a}}\right)$ & $\mathrm{LC}_{50}\left(95 \% \mathrm{CI}^{\mathrm{b}} ; \mathrm{mg} / \mathrm{L}\right)$ & $\chi^{2}(\mathrm{df})$ & $p$ Value & $R^{c}$ & No. & Slope (SE $\left.{ }^{\mathrm{a}}\right)$ & $\mathrm{LC}_{50}\left(95 \% \mathrm{CI}^{\mathrm{b}} ; \mathrm{mg} / \mathrm{L}\right)$ & $\chi^{2}(\mathrm{df})$ & $p$ Value & $\mathbf{R R}^{\mathrm{c}}$ \\
\hline HBCB-2019 & 270 & $2.10(0.26)$ & $1.17(0.92-1.45)$ & $0.91(3)$ & 0.82 & 7.80 & 270 & $2.49(0.32)$ & $3.31(2.32-4.22)$ & $1.36(3)$ & 0.82 & 6.62 \\
\hline HBWX-2019 & 270 & $2.42(0.28)$ & $0.27(0.21-0.33)$ & $0.63(3)$ & 0.89 & 1.80 & 270 & $2.31(0.28)$ & $2.47(1.90-3.03)$ & $3.10(3)$ & 0.38 & 4.94 \\
\hline HBJM-2019 & 270 & $2.64(0.32)$ & $0.83(0.62-1.03)$ & $1.80(3)$ & 0.61 & 5.53 & 270 & $2.86(0.32)$ & $2.86(2.33-3.39)$ & $2.50(3)$ & 0.48 & 5.72 \\
\hline HNNX-2019 & 270 & $2.32(0.27)$ & $1.26(1.01-1.52)$ & $0.99(3)$ & 0.80 & 8.40 & 270 & $2.22(0.26)$ & $8.08(6.61-10.21)$ & $0.73(3)$ & 0.86 & 16.16 \\
\hline JXNC-2019 & 270 & $1.65(0.26)$ & $2.53(1.75-4.69)$ & $1.76(3)$ & 0.62 & 16.87 & 270 & $1.73(0.23)$ & $7.51(5.91-9.89)$ & $8.31(3)$ & 0.04 & 15.02 \\
\hline AHLA-2019 & 270 & $1.66(0.23)$ & 1.49 (1.15-1.92) & $2.39(3)$ & 0.50 & 9.93 & 270 & $1.88(0.24)$ & $8.14(6.50-10.54)$ & $0.67(3)$ & 0.88 & 16.28 \\
\hline HNXY-2019 & 270 & $1.52(0.24)$ & $1.72(1.26-2.75)$ & $5.95(3)$ & 0.11 & 11.47 & 270 & $2.51(0.29)$ & $2.81(2.33-3.50)$ & $0.21(3)$ & 0.98 & 5.62 \\
\hline HBDY-2020 & 270 & $2.06(0.27)$ & $0.77(0.54-0.99)$ & $3.70(3)$ & 0.30 & 5.13 & 270 & $3.28(0.23)$ & $3.46(2.45-4.33)$ & $1.45(3)$ & 0.70 & 6.92 \\
\hline HBCB-2020 & 270 & $2.25(0.27)$ & $1.05(0.82-1.29)$ & 4.99 (3) & 0.17 & 7.00 & 270 & $1.89(0.24)$ & $3.17(2.46-3.96)$ & $2.19(3)$ & 0.53 & 6.34 \\
\hline HBWX-2020 & 270 & $2.53(0.28)$ & $0.44(0.37-0.54)$ & $4.47(3)$ & 0.22 & 2.93 & 270 & $1.55(0.23)$ & $7.08(5.34-10.45)$ & $1.32(3)$ & 0.73 & 14.16 \\
\hline HBJL-2020 & 270 & $1.69(0.25)$ & $1.89(1.40-3.01)$ & $2.92(3)$ & 0.40 & 12.60 & 270 & $1.88(0.25)$ & $7.01(5.50-9.71)$ & $3.16(3)$ & 0.37 & 14.02 \\
\hline HBJM-2020 & 270 & $2.51(0.28)$ & $1.29(1.06-1.55)$ & $1.90(3)$ & 0.59 & 8.60 & 270 & $4.08(0.46)$ & $6.39(5.44-7.34)$ & 3.09 (3) & 0.38 & 12.78 \\
\hline HBXZ-2020 & 270 & $1.96(0.25)$ & $2.28(1.83-2.99)$ & $3.46(3)$ & 0.33 & 15.20 & 270 & $2.09(0.25)$ & $8.44(6.84-10.47)$ & $2.36(3)$ & 0.50 & 16.88 \\
\hline HNNX-2020 & 270 & $2.02(0.25)$ & $1.19(0.92-1.47)$ & $2.37(3)$ & 0.50 & 7.93 & 270 & $2.32(0.27)$ & $5.92(4.72-7.19)$ & $2.83(3)$ & 0.42 & 11.84 \\
\hline JXNC-2020 & 270 & $2.12(0.25)$ & $1.97(1.60-2.47)$ & $2.17(3)$ & 0.54 & 13.13 & 270 & $1.81(0.24)$ & $6.52(5.02-8.22)$ & $1.72(3)$ & 0.63 & 13.04 \\
\hline AHHF-2020 & 270 & $1.94(0.25)$ & $2.07(1.66-2.66)$ & $1.65(3)$ & 0.65 & 13.80 & 270 & $2.25(0.27)$ & $5.79(4.57-7.06)$ & $2.03(3)$ & 0.57 & 11.58 \\
\hline HNXY-2020 & 270 & $1.74(0.23)$ & $1.79(1.38-2.28)$ & $2.64(3)$ & 0.45 & 11.93 & 270 & $2.47(0.29)$ & $2.35(1.80-2.87)$ & $3.13(3)$ & 0.37 & 4.70 \\
\hline GZQN-2020 & 270 & $1.39(0.24)$ & $2.60(1.73-5.35)$ & $1.63(3)$ & 0.65 & 17.33 & 270 & $1.77(0.25)$ & $16.13(12.30-23.74)$ & $0.77(3)$ & 0.86 & 32.26 \\
\hline
\end{tabular}


Table 3. Cont.

\begin{tabular}{|c|c|c|c|c|c|c|c|c|c|c|c|c|}
\hline \multicolumn{13}{|c|}{$\mathrm{D}$} \\
\hline \multirow{2}{*}{ Population } & \multicolumn{6}{|c|}{ Buprofezin } & \multicolumn{6}{|c|}{ Chlorpyrifos } \\
\hline & No. & Slope (SE ${ }^{\text {a) }}$ & $\mathrm{LC}_{50}\left(95 \% \mathrm{CI}^{\mathrm{b}} ; \mathrm{mg} / \mathrm{L}\right)$ & $\chi^{2}(\mathrm{df})$ & $p$ Value & $\mathbf{R R}^{\mathrm{c}}$ & No. & Slope (SE ${ }^{\text {a) }}$ & $\mathrm{LC}_{50}\left(95 \% \mathrm{CI}^{\mathrm{b}} ; \mathrm{mg} / \mathrm{L}\right)$ & $\chi^{2}(\mathrm{df})$ & $p$ Value & $\mathbf{R R}^{\mathrm{c}}$ \\
\hline НВСВ-2019 & 270 & $1.89(0.24)$ & $3.90(3.09-4.90)$ & $0.86(3)$ & 0.84 & 97.50 & 270 & $2.44(0.28)$ & $30.72(25.28-39.14)$ & $1.80(3)$ & 0.83 & 128.00 \\
\hline HBWX-2019 & 270 & $1.72(0.23)$ & $3.71(2.87-4.74)$ & $4.39(3)$ & 0.22 & 92.75 & 270 & $1.74(0.23)$ & $20.40(15.96-26.16)$ & $3.27(3)$ & 0.35 & 85.00 \\
\hline НВЈМ-2019 & 270 & $1.93(0.24)$ & $3.42(2.69-4.26)$ & $1.01(3)$ & 0.80 & 85.50 & 270 & $3.09(0.33)$ & $20.67(17.60-24.35)$ & $7.13(3)$ & 0.07 & 86.13 \\
\hline HNNX-2019 & 270 & $1.95(0.26)$ & $3.25(2.33-4.14)$ & $1.01(3)$ & 0.80 & 81.25 & 270 & $3.57(0.40)$ & $54.07(46.52-64.75)$ & $5.00(3)$ & 0.17 & 225.29 \\
\hline JXNC-2019 & 270 & $1.43(0.23)$ & $13.26(9.55-21.97)$ & $4.70(3)$ & 0.20 & 331.50 & 270 & $2.39(0.27)$ & $32.51(26.36-39.23)$ & $4.72(3)$ & 0.19 & 135.46 \\
\hline AHLA-2019 & 270 & $1.57(0.23)$ & $6.80(5.22-9.03)$ & $1.19(3)$ & 0.75 & 170.00 & 270 & $2.02(0.26)$ & $73.00(57.61-100.50)$ & $0.81(3)$ & 0.85 & 304.17 \\
\hline HNXY-2019 & 270 & $1.99(0.25)$ & $5.01(4.04-6.39)$ & $6.49(3)$ & 0.09 & 125.25 & 270 & $1.96(0.24)$ & $46.58(37.47-59.03)$ & $6.34(3)$ & 0.10 & 194.08 \\
\hline HBDY-2020 & 270 & $1.89(0.24)$ & $4.64(3.58-5.80)$ & $1.71(3)$ & 0.64 & 105.45 & 270 & $2.92(0.32)$ & $39.04(32.97-46.13)$ & $0.38(3)$ & 0.94 & 162.67 \\
\hline HBCB-2020 & 270 & $1.65(0.23)$ & $5.20(4.05-6.96)$ & $1.30(3)$ & 0.73 & 118.18 & 270 & $2.68(0.29)$ & $15.33(12.81-18.27)$ & $2.37(3)$ & 0.50 & 63.88 \\
\hline HBWX-2020 & 270 & $1.48(0.22)$ & $5.70(4.32-8.04)$ & $2.25(3)$ & 0.52 & 129.55 & 270 & $2.14(0.27)$ & $11.46(8.53-14.32)$ & $1.50(3)$ & 0.68 & 47.75 \\
\hline HBJL-2020 & 270 & $2.33(0.28)$ & $7.13(5.75-9.48)$ & $1.45(3)$ & 0.69 & 162.05 & 270 & $3.07(0.34)$ & $26.34(21.29-31.22)$ & $2.14(3)$ & 0.54 & 109.75 \\
\hline HBJM-2020 & 270 & $1.70(0.23)$ & $5.05(3.96-6.68)$ & $3.74(3)$ & 0.29 & 114.77 & 270 & $3.00(0.32)$ & $21.34(18.14-25.25)$ & $4.24(3)$ & 0.24 & 88.92 \\
\hline HBXZ-2020 & 270 & $1.45(0.23)$ & $6.60(4.94-9.77)$ & $0.34(3)$ & 0.95 & 150.00 & 270 & $2.36(0.31)$ & $43.69(34.31-61.90)$ & $1.74(3)$ & 0.63 & 182.04 \\
\hline HNNX-2020 & 270 & $1.57(0.23)$ & $4.43(3.23-5.76)$ & $3.57(3)$ & 0.31 & 100.68 & 270 & $3.20(0.34)$ & $39.65(33.81-46.46)$ & $2.47(3)$ & 0.48 & 165.21 \\
\hline JXNC-2020 & 270 & $1.59(0.23)$ & $5.51(4.18-7.16)$ & $0.69(3)$ & 0.87 & 137.75 & 270 & $2.06(0.25)$ & $22.88(18.55-28.69)$ & $1.34(3)$ & 0.72 & 95.33 \\
\hline AHHF-2020 & 270 & $1.58(0.23)$ & $3.88(2.76-5.07)$ & $2.49(3)$ & 0.48 & 88.18 & 270 & $2.27(0.26)$ & $45.34(37.32-55.83)$ & $0.30(3)$ & 0.96 & 188.92 \\
\hline HNXY-2020 & 270 & $1.51(0.22)$ & $4.30(3.27-5.73)$ & $1.37(3)$ & 0.71 & 97.73 & 270 & $1.68(0.24)$ & $39.41(29.88-58.46)$ & $1.49(3)$ & 0.69 & 164.21 \\
\hline GZQN-2020 & 270 & $1.98(0.27)$ & $8.82(6.76-13.01)$ & $3.18(3)$ & 0.36 & 200.45 & 270 & $2.45(0.28)$ & $18.54(15.289-22.31)$ & $1.46(3)$ & 0.69 & 77.25 \\
\hline \multicolumn{13}{|c|}{$\mathbf{E}$} \\
\hline \multirow{2}{*}{ Population } & \multicolumn{6}{|c|}{ Isoprocarb } & \multicolumn{6}{|c|}{ Ethofenprox } \\
\hline & No. & Slope $\left(S^{a}\right)$ & $\mathrm{LC}_{50}\left(95 \% \mathrm{CI}^{\mathrm{b}} ; \mathrm{mg} / \mathrm{L}\right)$ & $\chi^{2}(\mathrm{df})$ & $p$ Value & $\mathbf{R R}^{\mathrm{c}}$ & No. & Slope (SE $\left.{ }^{a}\right)$ & $\mathrm{LC}_{50}\left(95 \% \mathrm{CI}^{\mathrm{b}} ; \mathrm{mg} / \mathrm{L}\right)$ & $\chi^{2}(\mathrm{df})$ & $p$ Value & $\mathbf{R R}^{\mathrm{c}}$ \\
\hline НВСВ-2019 & 270 & $2.63(0.29)$ & 97.84 (81.69-116.95) & $0.88(3)$ & 0.83 & 10.39 & 270 & $1.25(0.22)$ & $435.50(304.77-770.96)$ & $0.53(3)$ & 0.91 & 12.58 \\
\hline HBWX-2019 & 270 & $2.16(0.26)$ & 91.57 (73.98-112.21) & $2.52(3)$ & 0.47 & 9.72 & 270 & $1.86(0.24)$ & $252.99(201.67-327.75)$ & $5.73(3)$ & 0.13 & 7.31 \\
\hline HBJM-2019 & 270 & $2.53(0.28)$ & $124.26(103.72-152.18)$ & $2.79(3)$ & 0.43 & 13.20 & 270 & $2.13(0.26)$ & $327.97(263.69-434.08)$ & $1.99(3)$ & 0.57 & 9.47 \\
\hline HNNX-2019 & 270 & $2.64(0.30)$ & 86.96 (73.33-104.62) & $4.89(3)$ & 0.18 & 9.23 & 270 & $1.62(0.25)$ & $575.55(420.53-933.75)$ & $1.91(3)$ & 0.59 & 16.63 \\
\hline JXNC-2019 & 270 & $1.43(0.24)$ & $215.22(150.02-390.68)$ & $0.04(3)$ & 0.99 & 22.85 & 270 & $1.38(0.22)$ & $209.86(154.59-322.82)$ & $2.46(3)$ & 0.48 & 6.06 \\
\hline AHLA-2019 & 270 & $1.65(0.23)$ & $75.78(58.24-97.76)$ & $1.52(3)$ & 0.68 & 8.04 & 270 & $1.61(0.24)$ & $275.60(202.47-440.60)$ & $0.53(3)$ & 0.91 & 7.96 \\
\hline HNXY-2019 & 270 & $1.88(0.24)$ & $26.14(20.86-33.95)$ & $4.80(3)$ & 0.19 & 2.78 & 270 & $2.14(0.26)$ & $256.60(209.20-324.06)$ & $0.63(3)$ & 0.89 & 7.41 \\
\hline HBDY-2020 & 270 & $1.70(0.24)$ & $134.86(104.16-190.17)$ & $2.07(3)$ & 0.56 & 14.32 & 270 & $1.27(0.22)$ & $301.41(219.77-458.33)$ & $0.73(3)$ & 0.87 & 8.71 \\
\hline HBCB-2020 & 270 & $2.23(0.26)$ & $68.42(55.22-83.31)$ & $0.64(3)$ & 0.89 & 7.26 & 270 & $2.26(0.26)$ & $232.67(191.46-287.45)$ & $2.46(3)$ & 0.48 & 6.72 \\
\hline HBWX-2020 & 270 & $2.32(0.27)$ & $116.69(96.33-143.72)$ & $2.00(3)$ & 0.57 & 12.39 & 270 & $1.33(0.22)$ & $311.79(229.35-470.59)$ & $2.85(3)$ & 0.41 & 9.01 \\
\hline HBJL-2020 & 270 & $2.04(0.25)$ & 140.85 (113.65-182.22) & $2.57(3)$ & 0.46 & 14.95 & 270 & $1.60(0.26)$ & $679.15(459.97-1333.14)$ & $3.75(3)$ & 0.29 & 19.62 \\
\hline
\end{tabular}


Table 3. Cont.

\begin{tabular}{|c|c|c|c|c|c|c|c|c|c|c|c|c|}
\hline \multicolumn{13}{|c|}{ E } \\
\hline \multirow{2}{*}{ Population } & \multicolumn{6}{|c|}{ Isoprocarb } & \multicolumn{6}{|c|}{ Ethofenprox } \\
\hline & No. & Slope (SE $\left.{ }^{a}\right)$ & $\mathrm{LC}_{50}\left(95 \% \mathrm{CI}^{\mathrm{b}} ; \mathrm{mg} / \mathrm{L}\right)$ & $\chi^{2}(\mathrm{df})$ & $p$ Value & $\mathbf{R R}^{\mathrm{c}}$ & No. & Slope (SE $\left.{ }^{a}\right)$ & $\mathrm{LC}_{50}\left(95 \% \mathrm{CI}^{\mathrm{b}} ; \mathrm{mg} / \mathrm{L}\right)$ & $\chi^{2}(\mathrm{df})$ & $p$ Value & $\mathbf{R R}^{\mathrm{c}}$ \\
\hline HBXZ-2020 & 270 & $2.25(0.28)$ & $171.83(138.58-227.68)$ & $6.35(3)$ & 0.10 & 18.24 & 270 & $1.17(0.21)$ & $268.36(191.17-410.57)$ & $5.27(3)$ & 0.15 & 7.75 \\
\hline HNNX-2020 & 270 & $1.89(0.25)$ & $120.27(95.57-160.13)$ & $1.06(3)$ & 0.79 & 12.77 & 270 & $1.10(0.22)$ & $561.88(362.20-1273.63)$ & $0.51(3)$ & 0.92 & 16.23 \\
\hline JXNC-2020 & 270 & $1.97(0.25)$ & 101.17 (81.44-129.51) & $3.98(3)$ & 0.26 & 10.74 & 270 & $1.54(0.23)$ & $232.41(173.47-353.93)$ & $2.45(3)$ & 0.49 & 6.72 \\
\hline AHHF-2020 & 270 & $2.13(0.26)$ & $60.14(47.36-73.85)$ & $1.34(3)$ & 0.72 & 6.38 & 270 & $1.34(0.22)$ & $226.23(167.47-330.85)$ & $0.64(3)$ & 0.89 & 6.54 \\
\hline GZQN-2020 & 270 & $1.98(0.26)$ & $183.31(144.14-253.85)$ & $1.42(3)$ & 0.70 & 19.46 & 270 & $1.71(0.27)$ & $665.53(458.41-1255.03)$ & $2.62(3)$ & 0.45 & 19.23 \\
\hline
\end{tabular}

${ }^{\text {a }} \mathrm{SE}$, standard error; ${ }^{\mathrm{b}} 95 \% \mathrm{CI}$, confidence interval; $\mathrm{df}$, degrees of freedom; ${ }^{\mathrm{c}} \mathrm{RR}$, resistance ratio. 


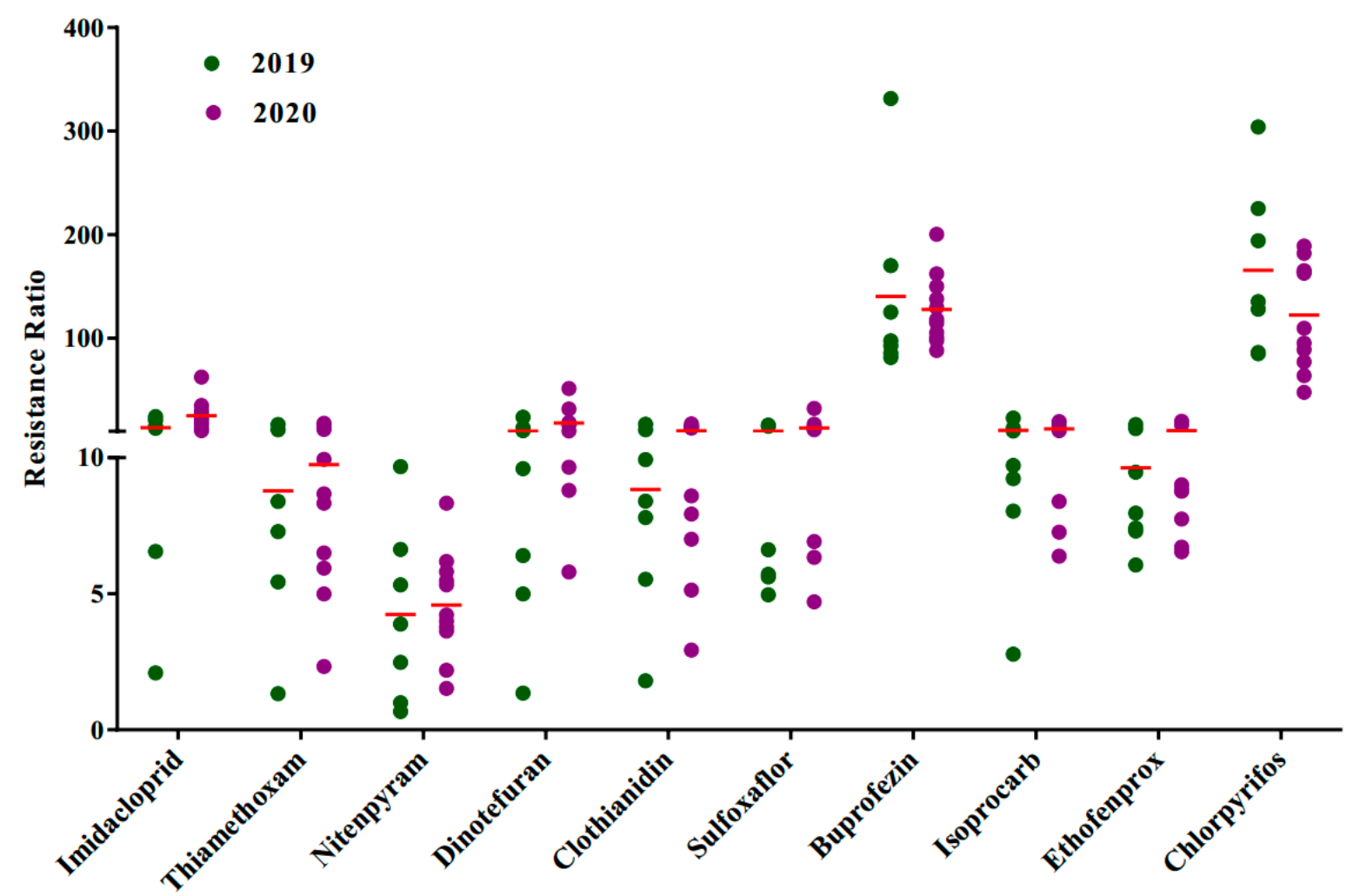

Figure 1. Resistance levels of field populations of $S$. furcifera to frequently used insecticides. Red horizontal lines across the scatter diagram represent the mean values of the resistance ratio of the different populations.

\subsection{Enzyme Activity}

To determine the role of detoxification enzymes in the insecticide resistance of S. furcifera, enzyme assays were performed to measure the levels of EST, GST and P450 (Figure 2A,B). The results showed that the EST activities from the populations of HNXY-2019 and HBCB2019 exhibited a 3.75-fold difference in 2019, while the maximum difference in 2020 was between the HBXZ-2020 population and the HBDY-2020 population, and there was a 3.30-fold difference. The GST activities from the populations of JXNC-2019 and HBCB-2019 resulted in 2.38-fold variation, while the GST activities of the population from AHHF-2020 were 1.60-fold higher than those of the HNNX-2020 population. The P450 activities resulted in a 2.60-fold variation between the populations from JXNC-2019 and HNXY-2019 in 2019 and a 3.53-fold variation between the populations from HNNX-2020 and HNXY-2020 in 2020.

\subsection{Pairwise Correlation Analysis}

To determine whether there were similar insecticidal patterns between insecticides, the logarithmic values of the $\mathrm{LC}_{50}$ measurements of the two insecticides were compared by pairwise correlation coefficients (Figure 3). Resistance to imidacloprid was significantly correlated with neonicotinoid insecticides, such as dinotefuran, thiamethoxam, clothianidin, nitenpyram and sulfoximine insecticide sulfoxaflor. Similarly, there were significant positive correlations between resistance to the insect growth regulator insecticides buprofezin and dinotefuran, thiamethoxam, clothianidin, sulfoxaflor, and isoprocarb. Moreover, significant correlations were found between isoprocarb and dinotefuran, sulfoxaflor and buprofezin.

In addition, we analyzed the correlation between the activity of three primary detoxification enzymes and the susceptibilities of S. furcifera to each insecticide (Figure 3). There were positive correlations between the $\mathrm{LC}_{50}$ values of nitenpyram, thiamethoxam and clothianidin and the activity of EST. In contrast, no significant correlations were found 
between the activities of glutathione $S$-transferase and the $\mathrm{LC}_{50}$ values of the insecticides, which was also the result for P450 activity.

A
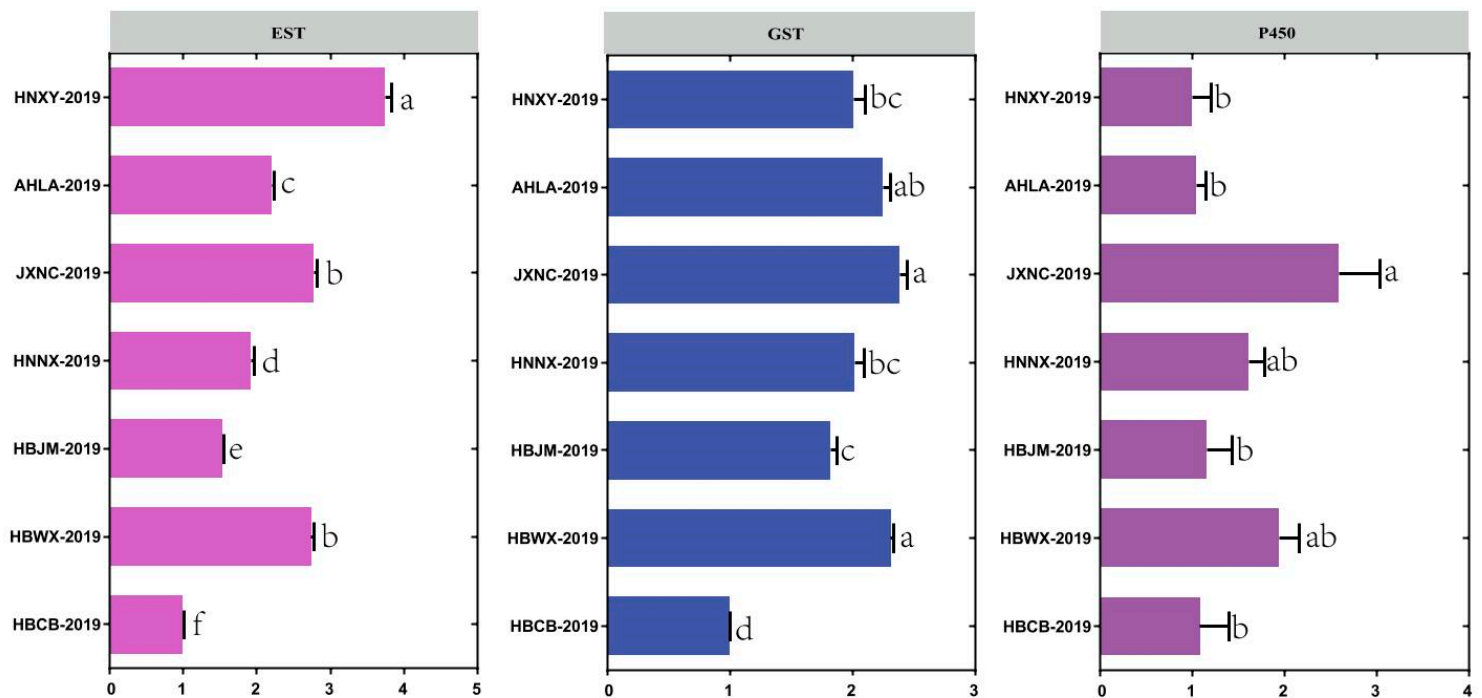

B
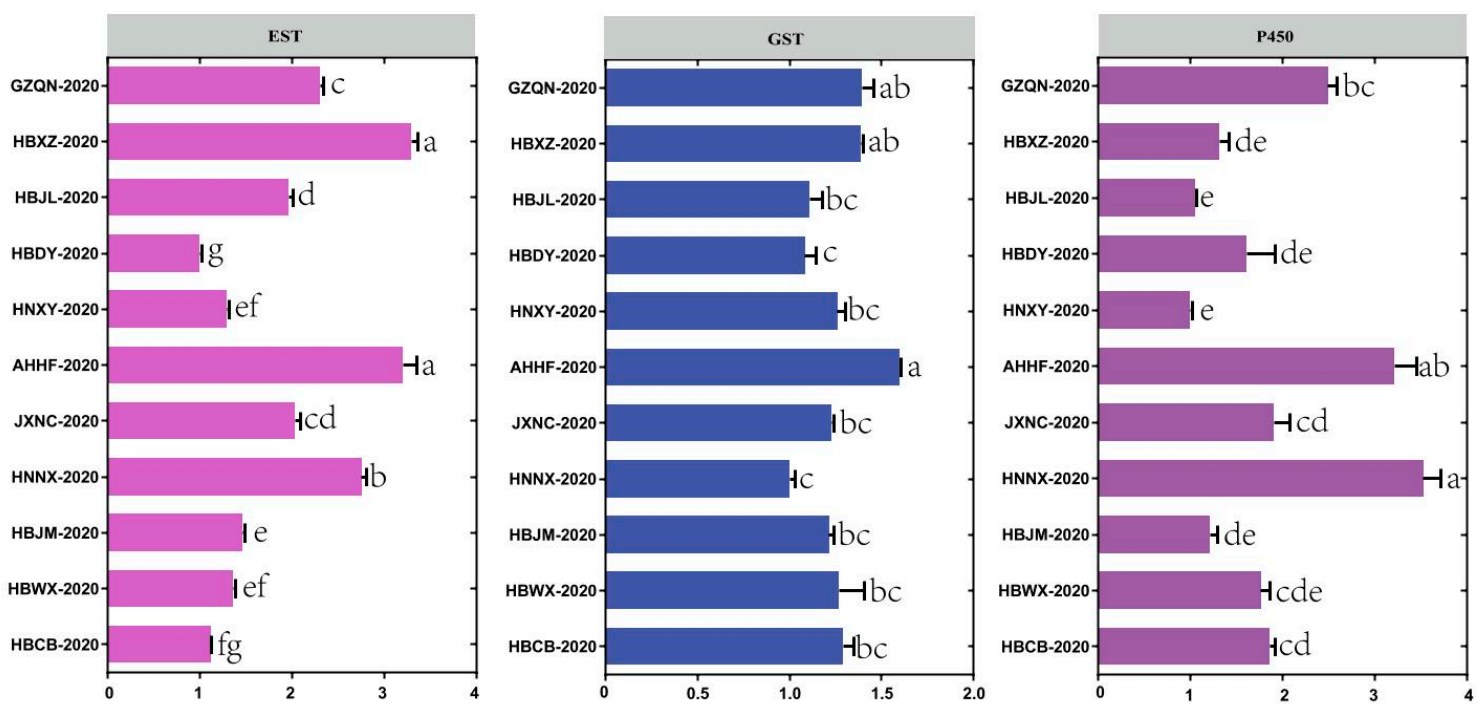

Figure 2. Relative activities of the detoxification enzymes in field populations of S. furcifera in 2019 (A) and 2020 (B). The relative enzyme activity of the detoxification enzyme corresponds to the ratio of the detoxification enzyme activity and the minimum enzyme activity. The SE bars with different small letters represent significant differences $(p<0.05)$. 


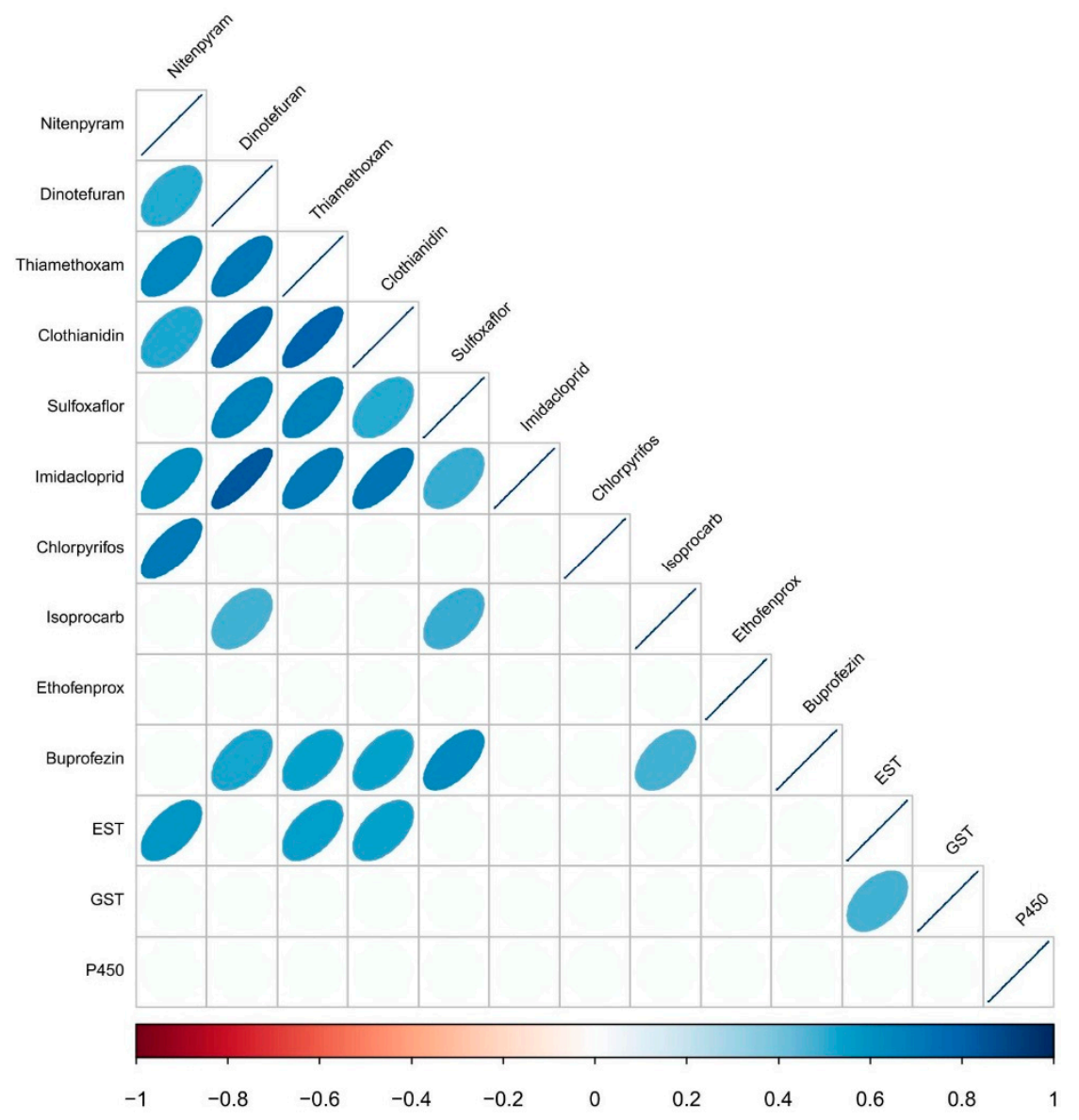

Figure 3. Correlations between the $\mathrm{LC}_{50}$ values of the tested insecticides and the enzyme activities of the field populations of $S$. furcifera. Correlations are based on linear Spearman correlation coefficients. The scale color of the filled squares indicates the strength of the correlation ( $r$ ) and whether it is negative (red) or positive (blue). Only significant correlations with $p<0.05$ are shown.

\section{Discussion}

Chemical insecticides remain the rational and primary tool for controlling pests [16,20-22]. Insecticide resistance monitoring is an important task for effective pest control and understanding insect resistance levels and biochemical resistance mechanisms to insecticides is the basis of integrated pest management (IPM) [23]. This study clarified the current status of the resistance of S. furcifera to 10 insecticides in six provinces of China from 2019 to 2020, which was of great value for the resistance monitoring and management of the pest.

Neonicotinoid insecticides are the most important chemical insecticides and are widely used to control various sucking pests [24,25]. Previous studies have suggested that S. furcifera has developed low to moderate resistance to neonicotinoid insecticides such as imidacloprid, nitenpyram, clothianidin, thiamethoxam and dinotefuran [26,27]. At present, the field population of $S$. furcifera still maintains low to moderate resistance to this type of insecticide, while the resistance level to some neonicotinoid insecticides showed an increasing trend in the period from 2019-2020. For instance, the resistance level of S. furcifera to imidacloprid in the AHHF-2020 population $(R R=62.55)$ nearly tripled compared with AHLA-2019 $(R R=24.27)$. The dramatic difference in imidacloprid resistance between these two fields suggests that resistance development to a compound can be area specific $[7,16]$. For other neonicotinoid insecticides, such as thiamethoxam, nitenpyram, dinotefuran and clothianidin, the resistance level of S. furcifera also increased slightly. However, the resistance of $S$. furcifera to nitenpyram and thiamethoxam was still maintained at low levels, indicating that it could be used interchangeably with other neonicotinoid insecticides. The above results indicated that neonicotinoid insecticides were still an important measure for 
farmers to control the whitebacked planthoppers. We recommend using compounds with different modes of action to delay the resistance to neonicotinoid insecticides.

Buprofezin, isoprocarb, ethofenprox and chlorpyrifos have been used to control S. furcifera with a long history in most rice-growing areas of southern Vietnam, China, Thailand and Malaysia [28-31]. Previous studies have suggested that S. furcifera has developed a high level of resistance to chlorpyrifos and buprofezin [2,32,33]. Similar to the results of previous studies, our results showed that $S$. furcifera still had a high level of resistance to chlorpyrifos and buprofezin [7]. We speculated that the long-distance migratory behavior of $S$. furcifera may be one of the reasons for this phenomenon. In addition, the resistance ratio of $S$. furcifera to isoprocarb and ethofenprox remained low, which may be due to the low frequency of use of the two insecticides in the monitored areas. Sulfoxaflor is a new type of sulfoximine insecticide that has high control efficacy on rice planthoppers [34-36]. Similar to the results in our previous study, S. furcifera developed moderate resistance levels to sulfoxaflor [7]. Studies have shown that resistance to sulfoxaflor was significantly correlated with neonicotinoid insecticides in N. lugens; therefore, we recommended that sulfoxaflor be used cautiously to control S. furcifera [37].

Enzyme activities can play a key role in understanding the patterns of resistance and insect susceptibility to chemicals [38]. Many previous reports found that P450, EST and GST were commonly the primary detoxification enzymes that help insect pests degrade a different type of xenobiotic [39-41]. Our results indicated that significant differences existed in the activity of detoxification enzymes between different populations. For instance, the EST activities were significantly different between the populations of JXNC-2019 and HNXY-2019, AHLA-2019, HNNX-2019, HBJM-2019 and HBCB-2019, which suggested that EST may be involved in the resistance of these populations to insecticides. Similarly, the P450 activities of AHHF-2020 and HNNX-2020 were higher than those of HBXZ-2020, HBJL-2020, HBDY-2020, HNXY-2020, JXNC-2020, HBJM-2020, HBWX-2020 and HBCB-2020, while enhancing P450 activity has been confirmed to be an important mechanism for the detoxification and metabolism of pests [42-44].

To delay or prevent the evolution of resistance, the most frequently used strategy is to alternate/rotate/mix pesticides [45]. Therefore, the cross-resistance between insecticides is worthy of attention. Previous studies have shown that significant cross-resistance exists between neonicotinoid insecticides in some pests. For instance, our results indicated that resistance to imidacloprid in S. furcifera was significantly associated with thiamethoxam, nitenpyram, dinotefuran, clothianidin and sulfoxaflor, which also occurred in small planthoppers, brown planthoppers, potato beetles, cotton aphids and peach aphids [37,46-49]. In addition, no risk of cross-resistance occurred between ethofenprox and neonicotinoid insecticides; therefore, it could be used as an important alternative insecticide for the control of whitebacked planthoppers. In our study, we found that the activity of EST was significantly correlated with nitenpyram, thiamethoxam clothianidin. Previous studies have shown that EST activities are related to the susceptibility of brown planthoppers to sulfoxaflor and nitenpyram $[37,39,49]$. Moreover, positively significant correlations were demonstrated between the activity of EST and clothianidin, nitenpyram and triflumezopyrim in field populations of $S$. furcifera [7]. These results suggested that EST might play an important role in the resistance of $S$. furcifera to nitenpyram, dinotefuran, clothianidin and imidacloprid.

\section{Conclusions}

Our findings revealed that field populations of whitebacked planthoppers have developed high levels of resistance to chlorpyrifos and buprofezin; therefore, we suggest that the use of chlorpyrifos and buprofezin should be suspended to control this pest. Additionally, although neonicotinoid insecticides are highly effective, the risk of cross-resistance should be given special attention. We recommend alternating the use of compounds with different modes of action that are more selectively compatible with natural enemies to slow down the resistance development of $S$. furcifera. Overall, this study provided useful data for the 
controlling of whitebacked planthoppers in the field, including the rational use of chemical insecticide, reducing the risk of control failure and input costs, which is in line with the definition of IPM.

Supplementary Materials: The following are available online at https:/ / www.mdpi.com/article/10 $.3390 /$ insects12121078/s1, Table S1: Concentrations of immersion for each insecticide.

Author Contributions: Conceptualization, H.W. and J.L.; data curation, Z.L. and Y.Q.; formal analysis, Z.L., Y.Q. and T.C.; funding acquisition, J.L.; investigation, Z.L., Y.Q., R.J., Y.Z., Z.R., C.Y., Y.L., Y.C. and Q.Z.; methodology, Z.L. and Y.Q.; project administration, H.W. and J.L.; resources, Z.L. and Y.Q.; software, Z.L., Y.Q. and T.C.; supervision, H.W. and J.L.; validation, Z.L. and Y.Q.; visualization, Z.L., Y.Q., Z.R., T.C., C.Y., Y.L., Y.C. and Q.Z.; writing-original draft, Z.L. and Y.Q.; writing-review and editing, Z.L., Y.Q., Y.Z., R.J., H.W. and J.L. All authors have read and agreed to the published version of the manuscript.

Funding: This work was supported by National Key Research and Development Program of China (2016YFD0200500) and National Science \& Technology Fundamental Resources Investigation Program of China (2018FY10100).

Institutional Review Board Statement: Not applicable.

Data Availability Statement: Not applicable.

Conflicts of Interest: The authors declare no conflict of interest.

\section{References}

1. Horgan, F.G.; Srinivasan, T.S.; Naik, B.S.; Ramal, A.F.; Bernal, C.C.; Almazan, M.L.P. Effects of nitrogen on egg-laying inhibition and ovicidal response in planthopper-resistant rice varieties. Crop Prot. 2016, 89, 223-230. [CrossRef] [PubMed]

2. Matsumura, M.; Sanada-Morimura, S.; Otuka, A.; Ohtsu, R.; Sakumoto, S.; Takeuchi, H.; Satoh, M. Insecticide susceptibilities in populations of two rice planthoppers, Nilaparvata lugens and Sogatella furcifera, immigrating into Japan in the period $2005-2012$. Pest Manag. Sci. 2014, 70, 615-622. [CrossRef]

3. Lei, W.; Liu, D.; Li, P.; Hou, M. Interactive effects of southern rice black-streaked dwarf virus infection of host plant and vector on performance of the vector, Sogatella furcifera (Homoptera: Delphacidae). J. Econ. Entomol. 2014, 107, 1721-1727. [CrossRef] [PubMed]

4. Pu, L.; Xie, G.; Ji, C.; Ling, B.; Zhang, M.; Xu, D.; Zhou, G. Transmission characteristics of Southern rice black-streaked dwarf virus by rice planthoppers. Crop Prot. 2012, 41, 71-76. [CrossRef]

5. Endo, S.; Nagata, T.; Kawabe, S.; Kazano, H. Changes of insecticide susceptibility of the white backed planthopper Sogatella furcifera Horvath (Homoptera:Delphacidae) and the brown planthopper Nilaparvata lugens Stal (Homoptera:Delphacidae). Appl. Entomol. Zool. 1988, 23, 417-421. [CrossRef]

6. Ping, X.; Endo, S.; Suzuki, K.; Ohtsu, K. The insecticide susceptibility of the brown planthopper, Nilaparvata lugens, and white-backed planthopper, Sogatella furcifera, collected from China and Japan. Kyushu Plant Prot. Res. 2001, 47, 54-57. [CrossRef]

7. Li, W.; Mao, K.; Liu, C.; Gong, P.; Xu, P.; Wu, G.; Le, W.; Wan, H.; You, H.; Li, J. Resistance monitoring and assessment of the control failure likelihood of insecticides in field populations of the whitebacked planthopper Sogatella furcifera (Horváth). Crop Prot. 2020, 127, 104973. [CrossRef]

8. Zhang, X.; Liao, X.; Mao, K.; Yang, P.; Li, D.; Ali, E.; Wan, H.; Li, J. Neonicotinoid insecticide resistance in the field populations of Sogatella furcifera (Horváth) in Central China from 2011 to 2015. J. Asia. Pac. Entomol. 2017, 20, 955-958. [CrossRef]

9. APRD. Arthropod Pesticide Resistance Database. 2021. Available online: http://www.pesticideresistance.org/search/Php/ (accessed on 12 August 2021).

10. Bass, C.; Denholm, I.; Williamson, M.S.; Nauen, R. The global status of insect resistance to neonicotinoid insecticides. Pestic. Biochem. Physiol. 2015, 121, 78-87. [CrossRef] [PubMed]

11. Benjamini, Y.; Hochberg, Y. Controlling the false discovery rate: A practical and powerful approach to multiple testing. J. R. Stat. Soc. Ser. B 1995, 57, 289-300. [CrossRef]

12. Sparks, T.C.; Nauen, R. IRAC: Mode of action classification and insecticide resistance management. Pestic. Biochem. Physiol. 2015, 121, 122-128. [CrossRef]

13. Ali, E.; Mao, K.; Liao, X.; Jin, R.; Li, J. Cross-resistance and biochemical characterization of buprofezin resistance in the whitebacked planthopper, Sogatella furcifera (Horvath). Pestic. Biochem. Physiol. 2019, 158, 47-53. [CrossRef]

14. Ruan, Y.; Wang, X.; Xiang, X.; Xu, X.; Guo, Y.; Liu, Y.; Yin, Y.; Wu, Y.; Cheng, Q.; Gong, C.; et al. Status of insecticide resistance and biochemical characterization of chlorpyrifos resistance in Sogatella furcifera (Hemiptera:Delphacidae) in Sichuan Province, China. Pestic. Biochem. Physiol. 2021, 171, 104723. [CrossRef] [PubMed] 
15. Ge, L.Q.; Huang, B.; Jiang, Y.P.; Gu, H.T.; Xia, T.; Yang, G.Q.; Liu, F.; Wu, J.C. Carboxylesterase precursor (EST-1) mediated the fungicide jinggangmycin-suppressed reproduction of Sogatella furcifera (Hemiptera: Delphacidae). J. Econ. Entomol. 2017, 110, 2199-2206. [CrossRef] [PubMed]

16. IRAC. Insecticide Resistance Action Committee. 2021. Available online: https://www.irac-online.org/modes-of-action/ (accessed on 25 October 2019).

17. Liao, X.; Mao, K.; Ali, E.; Zhang, X.; Wan, H.; Li, J. Temporal variability and resistance correlation of sulfoxaflor susceptibility among Chinese populations of the brown planthopper Nilaparvata lugens (Stål). Crop Prot. 2017, 102, 141-146. [CrossRef]

18. Fouad, E.A.; Abou-Yousef, H.M.; Abdallah, I.S.; Kandil, M.A. Resistance monitoring and enzyme activity in three field populations of cowpea aphid (Aphis craccivora) from Egypt. Crop Prot. 2016, 81, 163-167. [CrossRef]

19. Hangay, G.; Gayubo, S.F.; Hoy, M.A.; Goula, M.; Sanborn, A.; Morrill, W.L.; Gäde, G.; Marco, H.G.; Kabissa, J.C.B.; Ellis, J. Abbott's Formula. Encyclopedia of Entomology; Springer: Dordrecht, The Netherlands, 2008.

20. Aitio, A. A simple and sensitive assay of 7-ethoxycoumarin deethylation. Anal. Biochem. 1978, 85, 488-491. [CrossRef]

21. Lu, Y.; Wang, G.; Zhong, L.; Zhang, F.; Bai, Q.; Zheng, X.; Lu, Z. Resistance monitoring of Chilo suppressalis (Walker) (Lepidoptera: Crambidae) to chlorantraniliprole in eight field populations from east and central China. Crop Prot. 2017, 100, 196-202. [CrossRef]

22. Peng, Z.; Zheng, H.; Xie, W.; Wang, S.; Wu, Q.; Zhang, Y. Field resistance monitoring of the immature stages of the whitefly Bemisia tabaci to spirotetramat in China. Crop Prot. 2017, 98, 243-247. [CrossRef]

23. Sparks, T.C.; Crossthwaite, A.J.; Nauen, R.; Banba, S.; Cordova, D.; Earley, F.; Ebbinghaus-Kintscher, U.; Fujioka, S.; Hirao, A.; Karmon, D.; et al. Insecticides, biologics and nematicides: Updates to IRAC's mode of action classification-A tool for resistance management. Pestic. Biochem. Physiol. 2020, 167, 104587. [CrossRef] [PubMed]

24. Jeschke, P.; Nauen, R. Neonicotinoids-from zero to hero in insecticide chemistry. Pest Manag. Sci. 2008, 64, 1084-1098. [CrossRef]

25. Rao, Q.; Xu, Y.; Luo, C.; Zhang, H.; Jones, C.M.; Devine, G.J.; Gorman, K.; Denholm, I. Characterisation of neonicotinoid and pymetrozine resistance in strains of Bemisia tabaci (Hemiptera: Aleyrodidae) from China. J. Integr. Agric. 2012, 11, 321-326. [CrossRef]

26. Matsumura, M.; Sanada-Morimura, S.; Otuka, A.; Sonoda, S.; Van Thanh, D.; Van Chien, H.; Van Tuong, P.; Loc, P.M.; Liu, Z.W.; $\mathrm{Zhu}$, Z.R.; et al. Insecticide susceptibilities of the two rice planthoppers Nilaparvata lugens and Sogatella furcifera in East Asia, the Red River Delta, and the Mekong Delta. Pest Manag. Sci. 2018, 74, 456-464. [CrossRef]

27. Mu, X.C.; Zhang, W.; Wang, L.X.; Zhang, S.; Zhang, K.; Gao, C.F.; Wu, S.F. Resistance monitoring and cross-resistance patterns of three rice planthoppers, Nilaparvata lugens, Sogatella furcifera and Laodelphax striatellus to dinotefuran in China. Pestic. Biochem. Physiol. 2016, 134, 8-13. [CrossRef]

28. Endo, S.; Tsurumachi, M. Insecticide susceptibility of the brown planthopper and the white-backed planthopper collected from Southeast Asia. J. Pestic. Sci. 2001, 26, 82-86. [CrossRef]

29. Nagata, T. Monitoring on insecticide resistance of the brown planthopper and the white backed planthopper in Asia. J. Asia-Pac. Entomol. 2002, 5, 103-111. [CrossRef]

30. Nagata, T.; Masuda, T. Insecticide susceptibility and wing-form ratio of the brown planthopper, Nilaparvata lugens (Stål) (Hemiptera: Delphacidae) and the white backed planthopper, Sogatella furcifera (Horvath) (Hemiptera: Delphacidae) of Southeast Asia. Appl. Entomol. Zool. 1980, 15, 10-19. [CrossRef]

31. Su, J.; Wang, Z.; Zhang, K.; Tian, X.; Yin, Y.; Zhao, X.; Shen, A.; Gao, C.F. Status of insecticide resistance of the whitebacked planthopper, Sogatella furcifera (Hemiptera: Delphacidae). Florida Entomol. 2013, 96, 948-956. [CrossRef]

32. Kisimoto, R. Synoptic weather conditions inducing long-distance immigration of planthoppers, Sogatella furcifera Horvath and Nilaparvata lugens Stal. Ecol. Entomol. 1976, 1, 95-109. [CrossRef]

33. Nagata, T.; Kamimuro, T.; Wang, Y.C.; Han, S.G.; Noor, N.M. Recent status of insecticide resistance of long-distance migrating rice planthoppers monitored in Japan, China and Malaysia. J. Asia-Pac. Entomol. 2002, 5, 113-116. [CrossRef]

34. Cordova, D.; Benner, E.A.; Schroeder, M.E.; Holyoke, C.W.; Zhang, W.; Pahutski, T.F.; Leighty, R.M.; Vincent, D.R.; Hamm, J.C. Mode of action of triflumezopyrim: A novel mesoionic insecticide which inhibits the nicotinic acetylcholine receptor. Insect Biochem. Mol. Biol. 2016, 74, 32-41. [CrossRef]

35. Sparks, T.C.; Watson, G.B.; Loso, M.R.; Geng, C.; Babcock, J.M.; Thomas, J.D. Sulfoxaflor and the sulfoximine insecticides: Chemistry, mode of action and basis for efficacy on resistant insects. Pestic. Biochem. Physiol. 2013, 107, 1-7. [CrossRef]

36. Zhu, Y.; Loso, M.R.; Watson, G.B.; Sparks, T.C.; Rogers, R.B.; Huang, J.X.; Gerwick, B.C.; Babcock, J.M.; Kelley, D.; Hegde, V.B.; et al. Discovery and characterization of sulfoxaflor, a novel insecticide targeting sap-feeding pests. J. Agric. Food Chem. 2011, 59, 2950-2957. [CrossRef] [PubMed]

37. Liao, X.; Jin, R.; Zhang, X.; Ali, E.; Mao, K.; Xu, P.; Li, J.; Wan, H. Characterization of sulfoxaflor resistance in the brown planthopper, Nilaparvata lugens (Stål). Pest Manag. Sci. 2019, 75, 1646-1654. [CrossRef]

38. Hu, Z.; Feng, X.; Lin, Q.; Chen, H.; Li, Z.; Yin, F.; Liang, P.; Gao, X. Biochemical mechanism of chlorantraniliprole resistance in the diamondback moth, Plutella xylostella Linnaeus. J. Integr. Agric. 2014, 13, 2452-2459. [CrossRef]

39. Mao, K.; Ren, Z.; Li, W.; Cai, T.; Qin, X.; Wan, H.; Jin, B.R.; He, S.; Li, J. Carboxylesterase genes in nitenpyram-resistant brown planthoppers, Nilaparvata lugens. Insect Sci. 2021, 28, 1049-1060. [CrossRef]

40. Ullah, F.; Gul, H.; Tariq, K.; Desneux, N.; Gao, X.; Song, D. Functional analysis of cytochrome P450 genes linked with acetamiprid resistance in melon aphid, Aphis gossypii. Pestic. Biochem. Physiol. 2020, 170, 104687. [CrossRef] [PubMed] 
41. Yin, F.; Lin, Q.; Wang, X.; Li, Z.; Feng, X.; Shabbir, M.Z. The glutathione S-transferase (PxGST2L) may contribute to the detoxification metabolism of chlorantraniliprole in Plutella xylostella (L.). Ecotoxicology 2021, 30, 1007-1016. [CrossRef] [PubMed]

42. Bergé, J.B.; Feyereisen, R.; Amichot, M. Cytochrome P450 monooxygenases and insecticide resistance in insects. Philos. Trans. R. Soc. B Biol. Sci. 1998, 353, 1701-1705. [CrossRef] [PubMed]

43. Pan, Y.; Chai, P.; Zheng, C.; Xu, H.; Wu, Y.; Gao, X.; Xi, J.; Shang, Q. Contribution of cytochrome P450 monooxygenase CYP380C6 to spirotetramat resistance in Aphis gossypii Glover. Pestic. Biochem. Physiol. 2018, 148, 182-189. [CrossRef] [PubMed]

44. Zhang, Y.; Wang, Y.; Ma, Z.; Zhai, D.; Gao, X.; Shi, X. Cytochrome P450 monooxygenases-mediated sex-differential spinosad resistance in house flies Musca domestica (Diptera: Muscidae). Pestic. Biochem. Physiol. 2019, 157, 178-185. [CrossRef] [PubMed]

45. Roush, R.T. Designing resistance management programs: How can you choose? Pestic. Sci. 1989, 26, 423-441. [CrossRef]

46. Elzaki, M.E.A.; Zhang, W.; Han, Z. Cytochrome P450 CYP4DE1 and CYP6CW3v2 contribute to ethiprole resistance in Laodelphax striatellus (Fallén). Insect Mol. Biol. 2015, 24, 368-376. [CrossRef]

47. Kalsi, M.; Palli, S.R. Transcription factor cap n collar C regulates multiple cytochrome P450 genes conferring adaptation to potato plant allelochemicals and resistance to imidacloprid in Leptinotarsa decemlineata (Say). Insect Biochem. Mol. Biol. 2017, 83, 1-12. [CrossRef]

48. Ma, K.; Tang, Q.; Zhang, B.; Liang, P.; Wang, B.; Gao, X. Overexpression of multiple cytochrome P450 genes associated with sulfoxaflor resistance in Aphis gossypii Glover. Pestic. Biochem. Physiol. 2019, 157, 204-210. [CrossRef] [PubMed]

49. Mao, K.; Zhang, X.; Ali, E.; Liao, X.; Jin, R.; Ren, Z.; Wan, H.; Li, J. Characterization of nitenpyram resistance in Nilaparvata lugens (Stål). Pestic. Biochem. Physiol. 2019, 157, 26-32. [CrossRef] [PubMed] 